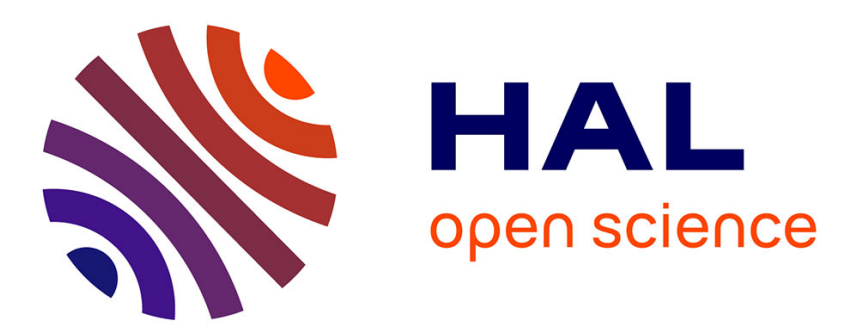

\title{
Inverse problem formulation for regularity estimation in images
}

Nelly Pustelnik, Patrice Abry, Herwig Wendt, Nicolas Dobigeon

\section{To cite this version:}

Nelly Pustelnik, Patrice Abry, Herwig Wendt, Nicolas Dobigeon. Inverse problem formulation for regularity estimation in images. International Conference on Image Processing (ICIP 2014), Oct 2014, Paris, France. pp. 6081-6085. hal-01399871

\section{HAL Id: hal-01399871 \\ https://hal.science/hal-01399871}

Submitted on 21 Nov 2016

HAL is a multi-disciplinary open access archive for the deposit and dissemination of scientific research documents, whether they are published or not. The documents may come from teaching and research institutions in France or abroad, or from public or private research centers.
L'archive ouverte pluridisciplinaire HAL, est destinée au dépôt et à la diffusion de documents scientifiques de niveau recherche, publiés ou non, émanant des établissements d'enseignement et de recherche français ou étrangers, des laboratoires publics ou privés. 


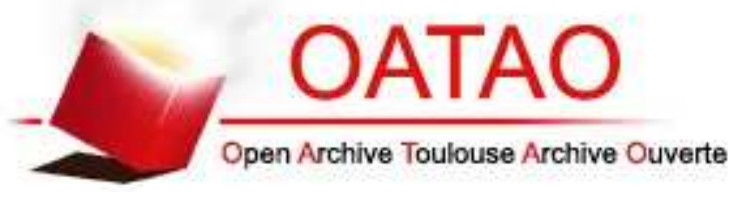

\section{Open Archive TOULOUSE Archive Ouverte (OATAO)}

OATAO is an open access repository that collects the work of Toulouse researchers and makes it freely available over the web where possible.

This is an author-deposited version published in : http://oatao.univ-toulouse.fr/ Eprints ID : 15172

The contribution was presented at ICIP 2014 :

https://icip2014.wp.mines-telecom.fr/

To cite this version : Pustelnik, Nelly and Abry, Patrice and Wendt, Herwig and Dobigeon, Nicolas Inverse problem formulation for regularity estimation in images. (2014) In: International Conference on Image Processing (ICIP 2014), 27 October 2014 - 30 October 2014 (Paris, France).

Any correspondence concerning this service should be sent to the repository administrator: staff-oatao@listes-diff.inp-toulouse.fr 


\title{
INVERSE PROBLEM FORMULATION FOR REGULARITY ESTIMATION IN IMAGES
}

\author{
Nelly Pustelnik ${ }^{1}$, Patrice Abry ${ }^{1}$, Herwig Wendt ${ }^{2}$ and Nicolas Dobigeon ${ }^{2}$ \\ ${ }^{1}$ Physics Dept. - ENSL, UMR CNRS 5672, F-69364 Lyon, France, firstname. lastnamedens-1yon. fr \\ ${ }^{2}$ IRIT, CNRS UMR 5505, INP-ENSEEIHT, F-31062 Toulouse, France, firstname. lastname@irit. fr
}

\begin{abstract}
The identification of texture changes is a challenging problem that can be addressed by considering local regularity fluctuations in an image. This work develops a procedure for local regularity estimation that combines a convex optimization strategy with wavelet leaders, specific wavelet coefficients recently introduced in the context of multifractal analysis. The proposed procedure is formulated as an inverse problem that combines the joint estimation of both local regularity exponent and of the optimal weights underlying regularity measurement. Numerical experiments using synthetic texture indicate that the performance of the proposed approach compares favorably against other wavelet based local regularity estimation formulations. The method is also illustrated with an example involving real-world texture.
\end{abstract}

Index Terms - Local regularity, variational approach, convex optimization, wavelet leaders

\section{INTRODUCTION}

Wavelet decompositions are now well recognized as sparsifying transforms and have been widely used in contexts of image compression and restoration $[1,2]$. Another very useful property of wavelet transforms found, so far, less widespread use in the field of image processing: their ability to evidence and measure scale invariance (cf., e.g., [3, 4, 5, 6, 7]). Notably, wavelet transforms have been shown to be relevant tools for the estimation of local regularity [5]. The earliest contribution to this subject traces back to [3], where the skeleton (maxima lines across scales) of the continuous wavelet transform is shown to enable practitioners to identify irregular (i.e., non-smooth) behavior in signals. By imposing an additional monotonicity constraint on the skeleton, the wavelet transform modulus maxima (WTMM) formalism has been further developed in, e.g., [4] to precisely measure Hölder exponents $h$. These constitute the canonical theoretical quantities for the measurement of local regularity and for performing multifractal analysis. More recently, it has been shown that accurate estimates of local regularity can be obtained using wavelet leaders (WL), specific multiresolution quantities that have been demonstrated to precisely reproduce Hölder exponents theoretically $[5,6]$. Wavelet leaders are defined as local suprema

This work is supported by the GdR 720 ISIS under the junior research project GALILEO and ANR AMATIS grant \#112432, 2010-2014. of the coefficients of the dyadic wavelet transform and inherit their computational efficiency, see, e.g., [6, 7] and Section 2 below for more precise definition.

Both the WTMM and the WL have been extensively used to perform multifractal analysis of real-world signals and images (see $[8,6,9,10]$ and references therein for examples of successful applications). In contrast to local regularity estimation, multifractal analysis does not aim at the time- or space-resolved estimation of $h$ but rather provides a global and geometric description of the fluctuations of $h$ in an image in terms of the so-called multifractal spectrum. However, for certain applications, the central information of interest is precisely the evolution along time or space of the Hölder exponent. In this case, multifractal analysis is not directly relevant. Instead, direct estimation of the local regularity evolution, sometimes referred to as multifractional analysis, needs to be performed. Yet, the estimation of $h$ at a precise time/space location suffers from poor performance that impairs its actual practical use. Consequently, the estimation of the time/space evolution of local regularity remains barely used in applications (see, a contrario, $[11,12])$. In particular, local estimation of regularity suffers from a large variance and common post-processing techniques for variance reduction, such as local smoothing, induce significant bias in the estimation of $h$ and inaccuracies in the localization of changes in regularity.

In a previous contribution [13], we have proposed an original two-step procedure that addresses the bias-variance tradeoff difficulty in the specific context of images with piece-wise constant regularity: (i) Unbiased estimation of the Hölder exponent for every position in the image using a patch-based wavelet leaders approach, (ii) Extraction of areas with uniform Hölder exponent from these local estimates using a variational procedure relying on total-variation (TV) [14]. In the present contribution, we elaborate on this approach and propose a one-step procedure that directly yields piece-wise constant local regularity estimates. The originality of the approach resides in combining estimation of $h$ and the TV denoising in a single-step. Through this formulation, an efficient local regularity estimation procedure is designed, yielding significantly increased estimation quality for images with piece-wise constant texture.

The remainder of this work is organized as follows. Section 2 recalls the notion of Hölder exponent, defines wavelet leaders and the state-of-the-art Hölder exponent estimation 
procedure. Section 3 formalizes the estimation of local regularity explicitly as an inverse problem and details the proposed variational procedure. Section 4 reports experimental results illustrating the performance of the proposed piecewise constant regularity estimation/segmentation procedure.

\section{HÖLDER EXPONENT AND WAVELET LEADERS}

Loosely speaking, the Hölder exponent $h(\underline{x})$ is a positive quantity that measures the regularity of a function at location $\underline{x} \in \mathbb{R}^{2}$ by comparing the evolution along scale of multiresolution quantities (cf. (2)) around $\underline{x}$ against a local power law behavior (see [5] for a precise definition). Qualitatively, a small value of the Hölder exponent indicates a locally highly irregular behavior, close to discontinuous, of the function, while a large value indicates local smoothness. A simple, efficient and theoretically well grounded solution to practically compute $h(\underline{x})$ relies on the use of wavelet leaders [6], which we define in what follows. Note that it has recently been proven [5] that wavelet leaders enable to measure the Hölder exponent for much more general classes of bi-dimensional functions than wavelet coefficients, and with a significantly improved accuracy [5, 6, 7, 13].

Let $f$ denote the bi-dimensional function taking bounded values (i.e, the image) to be analyzed. Let $\phi$ and $\psi$ denote respectively the scaling function and mother wavelet defining a 1D multiresolution analysis. The corresponding 2D tensor product wavelets are defined, for every $\underline{x}=\left(x_{1}, x_{2}\right) \in$ $\mathbb{R}^{2}$, as: $\psi^{(0)}(\underline{x})=\phi\left(x_{1}\right) \phi\left(x_{2}\right), \psi^{(1)}(\underline{x})=\psi\left(x_{1}\right) \phi\left(x_{2}\right)$, $\psi^{(2)}(\underline{x})=\phi\left(x_{1}\right) \psi\left(x_{2}\right)$, and $\psi^{(3)}(\underline{x})=\psi\left(x_{1}\right) \psi\left(x_{2}\right)$. The collection $\psi_{j, \underline{k}}^{(m)}(\underline{x})=2^{-j} \psi^{(m)}\left(2^{-j} \underline{x}-\underline{k}\right)$ of dilated (to scales $2^{j}$ ) and translated (to space positions $2^{j} \underline{k}$ ) templates of $\psi^{(m)}$ form a basis of $L^{2}\left(\mathbb{R}^{2}\right)$ for well chosen functions $\psi$. The $\left(L^{1}\right.$-normalized) discrete wavelet transform (DWT) coefficient at scale $j$, location $\underline{k}$ and subband $m \in\{1,2,3\}$ is defined as $d^{(m)}(j, \underline{k})=\left\langle f, 2^{-j} \psi_{j, \underline{k}}^{(m)}\right\rangle$.

The wavelet leader coefficient $L(j, \underline{k})$ is defined, for each scale $j$ and location $\underline{k}$, as the local supremum of all wavelet coefficients taken within a spatial neighborhood across all finer scales $j^{\prime} \leq j$

$$
L(j, \underline{k})=\sup _{\substack{m=\{1,2,3\} \\ \lambda_{j^{\prime}, \underline{k}^{\prime}} \subset \Lambda_{j, \underline{k}}}}\left|d^{(m)}\left(j^{\prime}, \underline{k}^{\prime}\right)\right|,
$$

where $\lambda_{j, \underline{k}}=\left[\underline{k} 2^{j},(\underline{k}+1) 2^{j}\right)$ and $\Lambda_{j, \underline{k}}=\bigcup_{p \in\{-1,0,1\}^{2}} \lambda_{j, \underline{k}+p}$ $[5,6]$. For every $\underline{x} \sim 2^{j} \underline{k}$, the wavelet leaders reproduce the Hölder exponent $h(\underline{x})$ as follows:

$$
L(j, \underline{k}) \simeq C(\underline{x}) 2^{j h(\underline{x})}
$$

when $2^{j} \rightarrow 0$ and where $C(\underline{x})$ denotes a constant. This relation can be rewritten as:

$$
\ln L(j, \underline{k}) \simeq j h(\underline{x})+\ln C(\underline{x}),
$$

which naturally leads to the use of linear regressions across scales at each location $\underline{k} \sim 2^{-j} \underline{x}$ for the estimation of $h(\underline{x})$, i.e.,

$$
\widehat{h}(\underline{x})=\sum_{j} w(j, \underline{k}) \ln L(j, \underline{k}) .
$$

Taking the expectations of (3) and (4) above yields

$$
\mathbb{E} \widehat{h}(\underline{x})=h(\underline{x}) \sum_{j} j w(j, \underline{k})+\ln C(\underline{x}) \sum_{j} w(j, \underline{k}),
$$

thus showing that the constraints

$$
\sum_{j} w(j, \underline{k}) \equiv 0 \quad \text { and } \quad \sum_{j} j w(j, \underline{k}) \equiv 1,
$$

ensure an unbiased estimation of $h$. Due to their local nature involving only a small number of coefficients, the estimates (4) have large variance. A straightforward attempt to reduce the variance consists in local spatial averaging (smoothing) of $h(\underline{x})$. However, local smoothing induces bias and prevents from accurately locating changes of $h$ in the image. To overcome this difficulty, a total variation (TV) optimization procedure, naturally favoring sharp edges, has been proposed in [13]. It requires the use of estimates of $h(\underline{x})$, which are obtained using (4) with fixed pre-defined weights $w(j, \underline{k})$. Although this approach yields relevant results, it does not enable to distinguish areas with Hölder exponents that are too close in value because the variance of (4) is too large.

In order to reduce the variance of (4), one can attempt to optimize the weights $w(j, \underline{k})$ which reflect the confidence granted to the quantities $\ln L(j, \underline{k})$. The variance of $\ln L(j, \underline{k})$ depends on the value of the Hölder exponent $h(\underline{x})$ at location $\underline{k} \sim 2^{-j} \underline{x}$ and the optimal choice of the weights $w(j, \underline{k})$ thus varies from one location to the other. Therefore, we propose in this article to formulate the estimation of $h(\underline{x})$ as an inverse problem involving jointly the Hölder exponents $h(\underline{x})$ and the weights $w(j, \underline{k})$ (constrained only by (6)) as parameters to be estimated. The inverse problem formulation and the proposed proximal based minimization procedure for finding its solution are detailed in the following section.

\section{INVERSE PROBLEM BASED LOCAL REGULARITY ESTIMATION}

"Degradation" model From now on, we make use of a discrete time formalism. Let $f=(f[n])_{1 \leq n \leq N}$ denote the vector representation of the image to be analyzed, of size $N=N_{1} \times N_{2}$. The orthonormal wavelet transform is labeled $F \in \mathbb{R}^{N \times N}$ and the wavelet coefficients of $f$ are denoted $d=(d[n])_{1 \leq n \leq N}=F f$. At each scale $j \in\{1, \ldots, J\}$, $\mathcal{L}_{j}: \mathbb{R}^{N} \rightarrow \mathbb{R}^{2^{-2 j} N}$ denotes the non-linear transform that associates the wavelet coefficients to the wavelet leaders $L_{j}=\left(L_{j}[k]\right)_{1 \leq k \leq 2-2 j N}$ (as defined in (1)) such that $L_{j}=\mathcal{L}_{j}(F f)$. A matrix formulation of (4) leads to:

$$
\sum_{j=j_{1}}^{j_{2}} W_{j} D_{j} \ln L_{j}=h+\varepsilon
$$


with $1 \leq j_{1}<j_{2} \leq J$ and where $\varepsilon$ models the uncertainties in the estimation, mostly due to the finite range of available scales. For each $j \in\left\{j_{1}, \ldots, j_{2}\right\}, W_{j} \in \mathbb{R}^{N \times N}$ denotes a diagonal matrix whose diagonal values are the regression weights, i.e, $W_{j}=\operatorname{diag}\left(w_{j}\right)$ with $w_{j}=\left(w_{j}[n]\right)_{1 \leq n \leq N} \in$ $\mathbb{R}^{N}$, and $D_{j} \in \mathbb{R}^{N \times 2^{-2 j} N}$ denotes a matrix that duplicates the signal such that, for every $(u, v) \in \mathbb{R}^{2^{-2 j} N} \times \mathbb{R}^{N}$, if we denote $\mathrm{u} \in \mathbb{R}^{2^{-j} N_{1} \times 2^{-j} N_{2}}$ (resp. $\mathrm{v} \in \mathbb{R}^{N_{1} \times N_{2}}$ ) the matrix representation of $u$ (resp. $v$ ), $v=D_{j} u$ means that, for every $\left(n_{1}, n_{2}\right) \in\left\{1, \ldots, N_{1}\right\} \times\left\{1, \ldots, N_{2}\right\}, \mathrm{v}\left[n_{1}, n_{2}\right]=$ $\mathrm{u}\left[\left\lceil 2^{-j} n_{1}\right\rceil,\left\lceil 2^{-j} n_{2}\right\rceil\right]$.

The model in (7) underlies an inverse problem in which $h=h([n])_{1 \leq n \leq N}$ need to be recovered from the logarithm of the wavelet leaders coefficients $\left(\ln L_{j}\right)_{j_{1} \leq j \leq j_{2}}$. This inverse problem resembles a denoising problem, yet including the additional challenge that a part of the observations (the regression weights matrices $\left(W_{j}\right)_{j_{1} \leq j \leq j_{2}}$ ) is unknown and must satisfy constraints (6).

Variational approach We propose to estimate the local regularity $h$ and the regression weight matrices $\left(W_{j}\right)_{j_{1} \leq j \leq j_{2}}$ by solving the following minimization problem:

$$
\begin{array}{r}
(\widehat{h}, \widehat{W}) \in \underset{h, W}{\operatorname{Argmin}}\left\|\sum_{j=j_{1}}^{j_{2}} W_{j} D_{j} \ln L_{j}-h\right\|_{2}^{2}+\lambda \mathrm{TV}(h) \\
+\eta_{1} \sum_{n=1}^{N} d_{C_{1}}(w[n])+\eta_{2} \sum_{n=1}^{N} d_{C_{2}}(w[n])
\end{array}
$$

where $W=\left(W_{j_{1}}, \ldots, W_{j_{2}}\right)$ and thus $w[n]=\left(w_{j_{1}}[n], \ldots\right.$, $\left.w_{j_{2}}[n]\right)$ belongs to $\mathbb{R}^{j_{2}-j_{1}+1}$. The first term denotes a data fidelity term. Distances to the convex sets $C_{1}$ and $C_{2}$, denoted $d_{C_{1}}$ and $d_{C_{2}}$, are introduced to provide some flexibility in the hyperplane constraints $C_{1}$ and $C_{2}$ :

$$
\begin{aligned}
& C_{1}=\left\{\left(\omega_{j_{1}}, \ldots, \omega_{j_{2}}\right) \in \mathbb{R} \times \ldots \times \mathbb{R} \mid \sum_{j=j_{1}}^{j_{2}} \omega_{j}=0\right\}, \\
& C_{2}=\left\{\left(\omega_{j_{1}}, \ldots, \omega_{j_{2}}\right) \in \mathbb{R} \times \ldots \times \mathbb{R} \mid \sum_{j=j_{1}}^{j_{2}} j \omega_{j}=1\right\} .
\end{aligned}
$$

For every $w \in \mathbb{R}^{\left(j_{2}-j_{1}+1\right)}, d_{C_{1}}(w)=\left\|w-P_{C_{1}}(w)\right\|$, with $P_{C_{1}}(w)=\arg \min _{v \in C_{1}}\|w-v\|^{2}$, denotes the projection onto the convex set $C_{1}$ (resp. $d_{C_{2}}$ and $P_{C_{2}}$ ). The second term $\operatorname{TV}(h)$ acts as a penalization that forces a solution with a minimal total variation [14] that is, for every $h \in \mathbb{R}^{N}$,

$$
\operatorname{TV}(h)=\|T h\|_{2,1}=\sum_{n=1}^{N} \sqrt{|(H h)[n]|^{2}+|(V h)[n]|^{2}}
$$

where $T=\left[H^{\top} V^{\top}\right]^{\top}$ with $H \in \mathbb{R}^{N \times N}$ and $V \in \mathbb{R}^{N \times N}$ are matrix representations of, respectively, the horizontal and vertical first-order discrete differences. The parameters $\lambda, \eta_{1}$, and $\eta_{2}$ will impact directly the solution. One could note that the choice of parameters $\lambda=1 / \eta_{1}=1 / \eta_{2}=0$ leads to the standard estimation procedure, formulated in (4) and (6).
Proximal algorithm The minimization problem (8) is convex but non-smooth. In the recent literature dedicated to nonsmooth convex optimization, several efficient algorithms have been proposed. For instance, when a Lipschitz data fidelity term is involved, such as a quadratic data fidelity term, as well as several regularization terms, such as TV regularization or distance to convex sets, one suited algorithm is referred to as CV (for Condat-Vũ) $[15,16]$. The corresponding iterations tailored to solve the problem in (8) are given in Algo. 1, that ensures convergence of the sequence $\left(h^{[\ell]}, W^{[\ell]}\right)_{\ell \in \mathbb{N}}$ to a solution of (8).

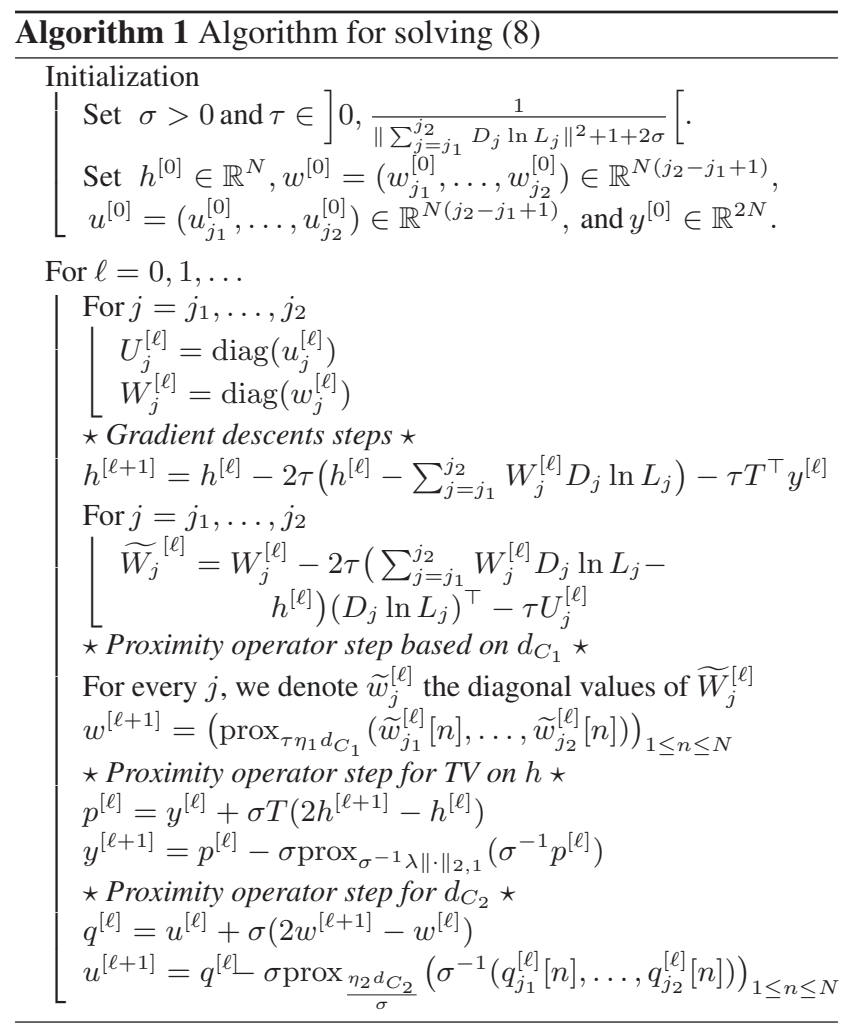

Algo. 1 requires the computation of the proximity operators associated to the mixed $\ell_{2,1}$-pseudo norm and to the distance to convex sets. Let us recall that the proximity operator associated to a convex, lower semi-continuous convex function $\varphi$ from $\mathcal{H}$ (where $\mathcal{H}$ denotes a real Hilbert space) to ]$-\infty,+\infty]$, denoted $\operatorname{prox}_{\varphi}$, is defined as, for every $u \in \mathcal{H}$, $\operatorname{prox}_{\varphi}(u)=\arg \min _{v \in \mathcal{H}} \frac{1}{2}\|u-v\|^{2}+\varphi(v)$. When $\varphi$ denotes the indicator function of a non-empty closed convex set $C \subset \mathcal{H}$, that is $\iota_{C}(x)=0$ if $x \in C$ and $+\infty$ otherwise, the proximity operator reduces to the projection, denoted $P_{C}$, onto the convex set.

The proximity operator steps involved in Algo. 1 have a closed-form expression. Indeed, it is shown in [17], that for every $u=(u[n])_{1 \leq n \leq N}$ with $u[n] \in \mathbb{R}^{2}$,

$$
\operatorname{prox}_{\frac{\lambda}{\sigma}\|\cdot\|_{2,1}} u=\left(\max \left(0,1-\frac{\lambda}{\sigma\|u[n]\|}\right) u[n]\right)_{1 \leq n \leq N}
$$




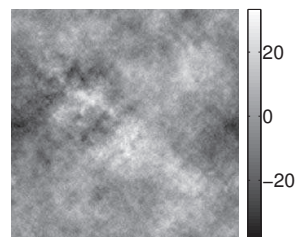

a) Original image $f$

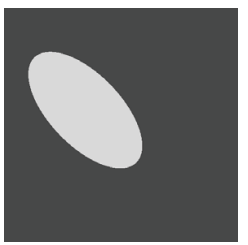

b) Regularity mask

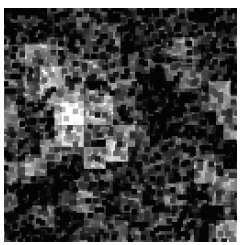

c) $\widehat{h}_{0}$ [7]

$(\mathrm{MSE}=0.275)$

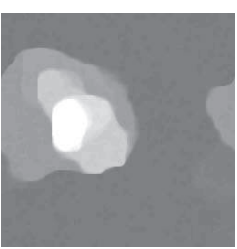

d) $\widehat{h}_{\lambda}[13]$

$(\mathrm{MSE}=0.052)$

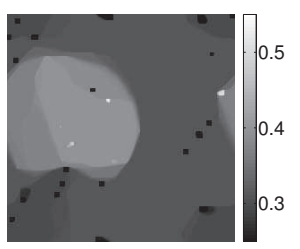

e) Proposed solution $(\mathrm{MSE}=0.025)$

Fig. 1. Simulated data

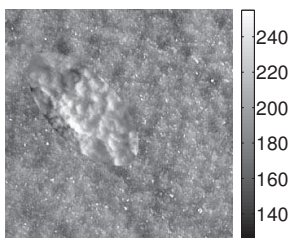

a) Original texture $f$

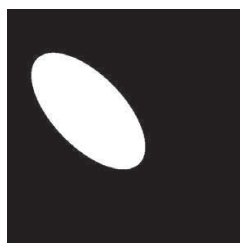

b) Texture mask White $=$ Cloud Black $=$ Snow

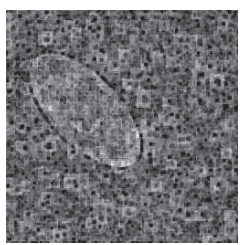

c) $\widehat{h}_{o}[7]$

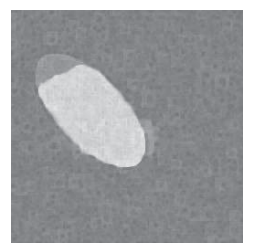

d) $\widehat{h}_{\lambda}[13]$

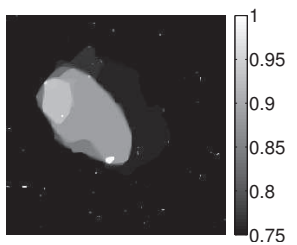

e) Proposed solution

Fig. 2. Real data

Moreover, according to [18, Proposition 2.8], if $C$ denotes a non-empty closed convex subset of $\mathbb{R}^{\left(j_{2}-j_{1}+1\right)}$ and if $\eta>0$, for every $u \in \mathbb{R}^{\left(j_{2}-j_{1}+1\right)}$,

$$
\operatorname{prox}_{\eta d_{C}} u=\left\{\begin{array}{lll}
u+\frac{\eta\left(P_{c}(u)-u\right)}{d_{C}(u)} & \text { if } & d_{C}(u)>\eta, \\
P_{C}(u) & \text { if } & d_{C}(u) \leq \eta .
\end{array}\right.
$$

For our purpose, $C$ models the hyperplane constraints $C_{1}$ and $C_{2}$ and one could note that $P_{C_{1}}$ and $P_{C_{2}}$ have a closed form expression given in [19].

\section{EXPERIMENTAL VALIDATION}

We first evaluate the performance of the proposed estimating strategy on synthetic data, consisting of $2 \mathrm{D}$ multifractional Brownian fields [20, 21], whose definition has been slightly modified here to ensure an homogeneous variance across the image (cf. [13] for details). The synthetic process has piece-wise constant regularity according to the mask shown in Fig. 1-b), with $h=0.5$ in the central area and $h=0.3$ for the background. A sample field is displayed in Fig. 1-a).

Analysis is conducted using a standard 2D DWT with orthonomal tensor product Daubechies mother wavelets with 2 vanishing moments. Regularity is estimated using the scaling range $\left(j_{1}, j_{2}\right)=(2,4)$. We compare the performance of the solution proposed against two other approaches. First, a standard estimation procedure with a priori fixed weights $w_{j}$ chosen to achieve ordinary linear regression, labelled $\widehat{h}_{o}$, (results are plotted in Fig. 1-c)). Second, we evaluate the performance of the technique proposed in [13] consisting in computing the proximity operator of the total variation of $\widehat{h}_{o}$, i.e, $\operatorname{prox}_{\lambda \mathrm{TV}}\left(\widehat{h}_{o}\right)$. The solution of this latter method is labelled $\widehat{h}_{\lambda}$ (cf., Fig. 1-d)) and the parameter $\lambda$ is empirically tuned to minimize the normalized mean square error (MSE) in this second approach. The solution proposed in Section 3, whose result is depicted in Fig. 1-e), achieves a smaller MSE and better evidence of the central area.

A second experiment deals with real data, obtained by mixing textures. The image is generated by inclusion of a distinct ellipse-shaped zone of cloud texture in a snow texture background (cf. Fig. 2-a)). First, it is worth noting that the unbiased local regularity estimation in Fig. 2-c) allows the edges of the added areas to be identified. However, without these synthetic edges, the local regularity change is difficult to identify. Similar observations are made for the solution obtained with [13], shown in Fig. 2-d) and the proposed solution. However, the proposed solution provides a better discrimination with sharper edges.

\section{CONCLUSION}

An efficient local regularity estimation strategy adapted to a multifractional framework has been devised. It enriches the classical estimation procedure by including the estimation of the weights that enter the linear regressions. These extra degrees of freedom yield local regularity estimates with significantly reduced variance. The proposed estimation procedure is formulated as an inverse problem and is solved using proximal minimization. Numerical experiments indicate that the proposed procedure significantly outperforms the standard estimation procedure and to further improve estimation performance compared to an earlier TV based procedure. Moreover, local regularity is also shown to be efficient to discriminate between visually similar textures such as cloud and snow. 


\section{REFERENCES}

[1] S. Mallat, A wavelet tour of signal processing, Academic Press, San Diego, USA, 1997.

[2] M. N. Do and M. Vetterli, "The contourlet transform: an efficient directional multiresolution image representation," IEEE Trans. Image Process., vol. 14, no. 12, pp. 2091-2106, Dec. 2005.

[3] S. Mallat and S. Zhong, "Characterization of signals from multiscale edges," IEEE Trans. Pattern Anal. Match. Int., vol. 14, no. 7, pp. 710-732, 1992.

[4] J.F. Muzy, E. Bacry, and A. Arnéodo, "Wavelets and multifractal formalism for singular signals: Application to turbulence data," Phys. Rev. Lett., vol. 67, no. 25, pp. 3515-3518, 1991.

[5] S. Jaffard, "Wavelet techniques in multifractal analysis," in Fractal Geometry and Applications: A Jubilee of Benoit Mandelbrot, Proceedings of Symposia in Pure Mathematics, M. Lapidus and M. van Frankenhuijsen, Eds. 2004, vol. 72, pp. 91-152, AMS.

[6] H. Wendt, P. Abry, and S. Jaffard, "Bootstrap for empirical multifractal analysis," IEEE Signal Process. Mag., vol. 24, no. 4, pp. 38-48, Jul. 2007.

[7] H. Wendt, S.G. Roux, P. Abry, and S. Jaffard, "Wavelet leaders and bootstrap for multifractal analysis of images," Signal Proces., vol. 89, pp. 1100-1114, 2009.

[8] A. Arneodo, N. Decoster, and S.G. Roux, "Intermittency, log-normal statistics, and multifractal cascade process in high-resolution satellite images of cloud structure," Phys. Rev. Lett., vol. 83, no. 6, pp. 12551258, 1999.

[9] A. Echelard and J. Lévy Véhel, "Self-regulating processes-based modeling for arrhythmia characterization," in Imaging and Signal Processing in Health Care and Technology, Baltimore, United States, May 2012.

[10] P. Abry, S. Jaffard, and H. Wendt, "When Van Gogh meets Mandelbrot: Multifractal classification of painting's texture," Signal Process., vol. 93, no. 3, pp. 554$572,2013$.

[11] B. Pesquet-Popescu and J. Lévy Vehel, "Stochastic fractal models for image processing," IEEE Signal Process. Mag., vol. 19, no. 5, pp. 48-62, Sep. 2002.

[12] O. Pont, A. Turiel, and H. Yahia, "An optimized algorithm for the evaluation of local singularity exponents in digital signals," in Combinatorial Image Analysis, J.K. Aggarwal et al., Ed., vol. 6636 of Lecture Notes in Computer Science, pp. 346-357. Springer Berlin Heidelberg, 2011.
[13] N. Pustelnik, H. Wendt, and P. Abry, "Local regularity for texture segmentation: Combining wavelet leaders and proximal minimization," in Proc. Int. Conf. Acoust., Speech Signal Process., Vancouver, Canada, May 2013, pp. 5348-5352.

[14] L. Rudin, S. Osher, and E. Fatemi, "Nonlinear total variation based noise removal algorithms," Physica D, vol. 60, no. 1-4, pp. 259-268, Nov. 1992.

[15] L. Condat, "A primal-dual splitting method for convex optimization involving Lipschitzian, proximable and linear composite terms," J. Optim. Theory Appl., vol. 158, no. 2, pp. 460-479, 2013.

[16] B. C. Vũ, "A splitting algorithm for dual monotone inclusions involving cocoercive operators," Adv. Comput. Math., vol. 38, pp. 667-681, 2011.

[17] G. Peyré and J. Fadili, "Group sparsity with overlapping partition functions," in Proc. Eur. Sig. Proc. Conference, Barcelona, Spain, Aug. 29 - Sept. 2, 2011, pp. 303-307.

[18] P. L. Combettes and J.-C. Pesquet, "A proximal decomposition method for solving convex variational inverse problems," Inverse Problems, vol. 24, no. 6, pp. 065014 , Dec. 2008.

[19] S. Theodoridis, K. Slavakis, and I. Yamada, "Adaptive learning in a world of projections," IEEE Signal Process. Mag., vol. 28, no. 1, pp. 97-123, Jan. 2011.

[20] A. Benassi, S. Jaffard, and D. Roux, "Gaussian processes and pseudo-differential elliptic operators," Rev. mat. Iberoamericana, vol. 8, no. 1, pp. 19-89, 1997.

[21] A. Ayache, S. Cohen, and J. Lévy Vehel, “The covariance structure of multifractional Brownian motion, with application to long range dependence," in Proc. Int. Conf. Acoust., Speech Signal Process., Dallas, Texas, USA, Mar. 14-19, 2000, pp. 3810-3813. 